\title{
Detection of active transposable elements in Arabidopsis thaliana using Oxford Nanopore Sequencing technology
}

\author{
Emilie Debladis ${ }^{1,2+}$, Christel Llauro ${ }^{1,2+}$, Marie-Christine Carpentier ${ }^{1,2}$, Marie Mirouze ${ }^{1,3}$ and Olivier Panaud ${ }^{1,2,4^{*}}$
}

\begin{abstract}
Background: Transposables elements (TES) contribute to both structural and functional dynamics of most eukaryotic genomes. Because of their propensity to densely populate plant and animal genomes, the precise estimation of the impact of transposition on genomic diversity has been considered as one of the main challenges of today's genomics. The recent development of NGS (next generation sequencing) technologies has open new perspectives in population genomics by providing new methods for high throughput detection of Transposable Elements-associated Structural Variants (TEASV). However, these have relied on Illumina platform that generates short reads (up to 350 nucleotides). This limitation in size of sequence reads can cause high false discovery rate (FDR) and therefore limit the power of detection of TEASVs, especially in the case of large, complex genomes. The newest sequencing technologies, such as Oxford Nanopore Technologies (ONT) can generate kilobases-long reads thus representing a promising tool for TEASV detection in plant and animals.

Results: We present the results of a pilot experiment for TEASV detection on the model plant species Arabidopsis thaliana using ONT sequencing and show that it can be used efficiently to detect TE movements. We generated a $\sim 0.8 \mathrm{X}$ genome coverage of a met1-derived epigenetic recombinant inbred line (epiRIL) using a Minlon device with R7 chemistry. We were able to detect nine new copies of the LTR-retrotransposon Evadé (EVD). We also evidenced the activity of the DNA transposon CACTA, CAC1.

Conclusions: Even at a low sequence coverage (0.8X), ONT sequencing allowed us to reliably detect several TE insertions in Arabidopsis thaliana genome. The long read length allowed a precise and un-ambiguous mapping of the structural variations caused by the activity of TEs. This suggests that the trade-off between read length and genome coverage for TEASV detection may be in favor of the former. Should the technology be further improved both in terms of lower error rate and operation costs, it could be efficiently used in diversity studies at population level.
\end{abstract}

Keywords: Transposable elements, Arabidopsis, Oxford Nanopore Technology, Sequencing

\section{Background}

Transposable elements (TEs) are widespread in eukaryotic genomes. Because of their nature and ability to increase in copy number, TEs play a major role in the structure and evolution of the genome in most

\footnotetext{
*Correspondence: panaud@univ-perp.fr; http://www.panaudlab.org

${ }^{\dagger}$ Equal contributors

${ }^{1}$ Université de Perpignan Via Domitia, Laboratoire Génome et Développement des Plantes, 52, avenue Paul alduy, 66860 Perpignan cedex, France

${ }^{2}$ Centre National de la Recherche Scientifique, Laboratoire Génome et Développement des Plantes, 52, avenue Paul alduy, 66860 Perpignan cedex, France

Full list of author information is available at the end of the article
}

eukaryotic lineages $[1,2]$. Transposable Elementsassociated structural variants (TEASVs) are common in natural and domesticated populations and contribute to a large extent to their genomic diversity, while they also can influence gene expression $[3,4]$ and lead to phenotypic variations $[5,6]$. Therefore, the estimation of the contribution of TEASVs to biological diversity in plant and animals has become one of the objectives of genomics today.

In the past decade, the emergence of Next Generation Sequencing (NGS) technologies based on Illumina platform has opened new perspectives for the development 
of new methods to conduct systematic genome-wide surveys of TEASVs. Several methods have been proposed for the detection of TEASVs using this technology $[7,8]$. These are referred to as paired-end mapping (PEM), depth of coverage (DOC) and split-read mapping (SRM). PEM is based on the mapping of both ends of amplicons from Illumina libraries onto a reference genome. Structural variants are detected when both ends of an amplicon map at different locations in the genome (i.e. at a distance which is significantly larger than the average size of the inserts of the Illumina library). The PEM was developed in order to best exploit the short reads that are paired from the same amplicon. In plants, this method allowed the detection of active TEs in rice $[9,10]$ and Arabidopsis thaliana [11]. In the latter case, the authors identified a LTR-retrotransposon Évadé $(E V D)$ which is active in an hypomethylated mutant plant. However, several authors pointed out that the mapping of short reads in complex genomes is not always reliable and can lead to high False Discovery Rate (FDR, see [12, 13]). This is mostly due to their presence at high copy number in genomes, thus challenging their detection in repeated regions. The DOC method consists in estimating the sequencing coverage of known TE families to eventually detect an increase in their copy number as a signature of transposition. It is therefore restricted to TEs that transpose via a copy and paste mechanism, such as LTR retrotransposons, LINEs or SINEs. The DOC method is very robust and can detect insertions in repeated regions, unlike the previous methods described above [14, 15]. However, its major limitation is that it does not provide any information regarding the position of new insertions.

The SRM method is based on the identification of sequencing reads that span the junction between a new TE insertion and its native insertion site. It is conceptually the most reliable methods for TEASV detection, but the short read length produced by the Illumina technology has so far impeded its reliable use for exhaustive TEASV detection. The recent improvement of the technology in this matter has however led some authors to successfully use the SRM method [16-18], but the short read length still does not allow any reliable detection of TE insertions in repeated sequences. The recent development of third generation technologies that allow the sequencing of a single molecule and can generate long reads could open new perspectives for TEASV detection through SRM : the two platforms available so far, i.e. Pacific Biosciences and Oxford Nanopore Technologies, indeed allow to generate kilobases-long reads that could improve the reliability of TEASVs detection by providing enough sequence information to unambiguously map new TE insertion sites.
The Nanopore technology was conceptualized three decades ago [19] but lead only five years ago to the commercial release of the smallest hand-held sequencers, called MinIon. This new Nanopore sequencer is based on DNA characteristics where the use of a positive electrical potential lead to an ionic current. As DNA molecules are negatively charged, they can translocate through a protein nanopore leading to changes in current that are used to determine the nucleotide base sequence [20,21]. So far, both Pacific Biosciences and Nanopore technologies have suffered from high sequencing error rate, which impedes the direct use of the sequencing data for genome assemblies. However, in the case of TEASV detection, the tradeoff between sequencing length and high error rate may be in favor of the former since we anticipate that a read of several kilobases could be unambiguously mapped onto a reference genome even if sequencing is achieved with an error rate of $10-15 \%$. In this report, we tested this hypothesis by sequencing the genome of a partially hypomethylated line of the plant species $A$. thaliana $[11,22]$ in which the LTR retrotransposon $E V D$ is transpositionally active. We indeed show that new insertions of $E V D$ as well as of another TE family could easily and reliably be detected in this plant material.

\section{Results}

\section{Characteristics of the raw dataset}

A qPCR assay of the epiRIL12 line that we used in this study suggested the presence of newly transposed copies of the retrotransposon $E V D$ (see Methods). This material was therefore perfectly suitable to tentatively detect these insertions using ONT sequencing platform. Three flow cells were used to sequence the genomic DNA of epiRIL12 line with two MinIon devices : one of the first available version and the other two of the MkI release type. The first MinIon run (FLO-MAP003) generated 49,061 sequence reads totalling $232 \mathrm{Mb}$. The ONT platform produces three types of reads : the template reads are produced from the forward strand sequencing. The complement reads are produced from the reverse strand sequencing. This distinction is made possible by the linking of the two strands of the molecule to be sequenced. The first strand to be sequenced is defined as the forward strand, then the second strand to be sequenced (from the same pore because it is physically linked to the first strand) is defined as the reverse strand. When both forward and complement reads are available for the same molecule, then the two sequences are merged into a single one, referred to as two direction reads or All 2D. For the first run, $98 \%$ of the molecules produced Template reads, while $40 \%$ produced both template and complement reads. Finally, 35\% produced All 2D reads merged into a single consensus sequence. The last two runs (FLO-MAP103) produced 9,796 and 
4,644 reads (66 and $38 \mathrm{Mb}$ ) respectively. 100\% were template reads, with 6,437 and 3,008 complement reads, respectively. Of these, a total of 5,490 and 2,357 were converted into All $2 \mathrm{D}$ reads.

Because the reads obtained from the 3 MinIon sequencing runs corresponded to the same DNA sample of epiRIL12, all sequence data were concatenated into a final multifasta file containing 118,554 sequences. The read length varied between 6 and 691,915 nucleotides, although $72 \%$ of the reads had a length comprised between 500 and 15,000 nucleotides with a median size of $4.6 \mathrm{~kb}$ (Additional file 1: Figure S2).

All reads were then mapped onto the Arabidopsis reference genome TAIR10 (The Arabidopsis Information Resource, http://www.arabidopsis.org) using blastN programme (see material and methods) with allowed gaps. 47,765 reads $(40.3 \%)$ produced a significant alignment and were kept for further analyses. These represent $\sim 80 \mathrm{Mbp}$ of sequences, i.e. $\sim 0.8 \mathrm{X}$ genome coverage. In order to validate this estimation, we searched for the presence of 10 selected unique Arabidopsis genes in our dataset and found that 8 produced a significant alignment with at least one of the 47,765 reads (Additional file 2: Figure S3), which confirms the first estimation. The average sequence identity between the reads and the reference Arabidopsis genome sequence was $85.8 \%$, which is close to the manufacturer's claim with the R7 chemistry (i.e. $88 \%$ ).

\section{Detection of EVD insertions sites}

The first step of our analysis was to search for all EVD insertions in the genome of the epiRIL12 line. For this, a simple similarity search of the EVD reference sequence used as a query against a database composed of 47,765 reads dataset, using the blastN programme was performed. Sixteen reads ranging from 4.2 to $8.8 \mathrm{~kb}$ showed similarity with EVD. Four of these reads entirely spanned the element (i.e. 100\% of the reads corresponded to the element). Eleven reads showed partial similarity to $E V D$, with one part of the sequence corresponding to the element and the other to another locus in the Arabidopsis genome, which corresponded to nine distinct insertion sites. One among these nine insertion sites corresponded to the native copy of EVD present on the chromosome 5 . Finally, one $7 \mathrm{~kb}$ read spanned the complete EVD sequence flanked by both insertion site sequences (Fig. 1). To our knowledge, this is the first example of the identification of a complete LTRretrotransposon insertion in a single sequencing read. The map positions of the nine newly inserted EVD elements are represented in Fig. 2.

A wet-lab validation of $E V D$ neo-insertion sites detected above was performed through PCR amplification and sequencing. All primers combinations confirmed the presence of the insertion at the expected location (Fig. 3 a to i). Five neoinsertions were found to be heterozygous (neo1, neo2,neo4, neo6 and neo8), while the remaining four are homozygous.

\section{Detection of transposition events ab initio}

The long reads generated by the ONT platform should theoretically allow a reliable detection of TEASVs using the SRM method. Our method is based on blastN similarity search rather than on Burrows-Wheeler based

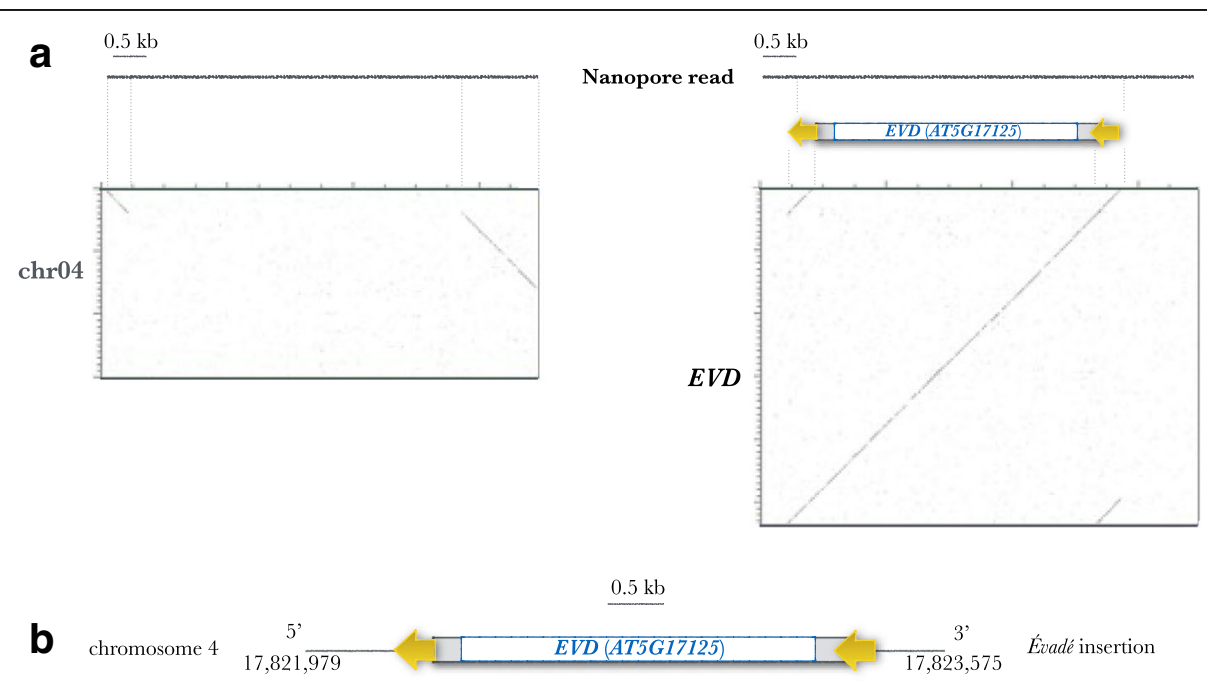

Fig. 1 Characterization of EVD neo-insertion 4. a Dot-plots comparison of a $7 \mathrm{~kb}$ Nanopore read (horizontal) with the region of the insertion on chromosome 4 (left, vertical) and the sequence of EVD (right, vertical). Blue box : GAG-POL region, grey boxes : UTR and yellow arrows : long terminal repeats. $\mathbf{b}$ Schematic representation of EVD insertion in the chromosome 4 . The orientation of the EVD LTRs are indicated by yellow arrows, the EVD insertion is in antisense 


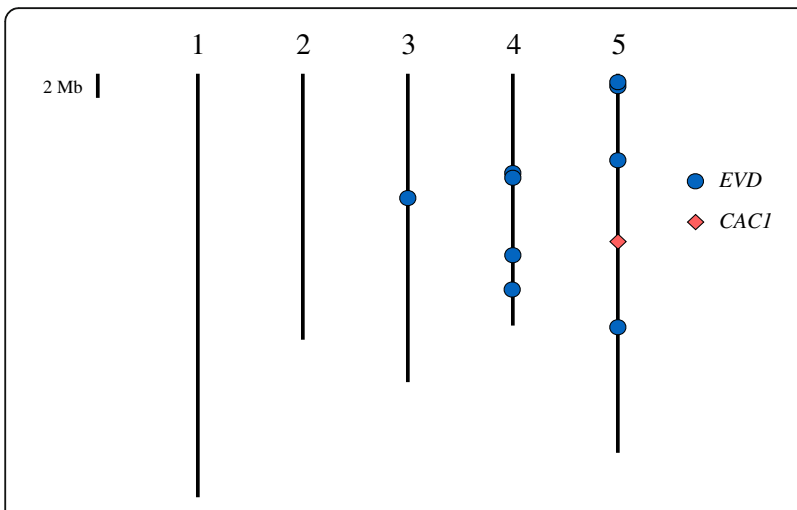

Fig. 2 Map position of all neo-insertions detected in this study. The vertical bars represent the five chromosomes of Arabidopsis thaliana. The length of each chromosome is proportional to its physical size

algorithms usually used with Illumina data. BW-based algorithms are designed for the mapping of a high number of short reads onto a reference genome. Moreover, BW mapping is usually achieved at high stringency, given the short read length. The Nanopore reads are, on the contrary, very long and exhibit a lot of mismatchs and indels, due to the high error rate. We therefore found the BLAST algorithm more appropriate for the analyses. Thirty-six MinIon reads were identified as spanning a similarity breakpoint and homologous to one sequence of the Arabidopsis TE database. These putative transposition events were carefully checked manually using simple dotter alignments of the reads against both the TE sequence and the flanking sequence of the insertion, in order to eliminate false positives (see the discussion section). Only 10 reads were thus validated. Nine of these insertion sites corresponded to $E V D$ transposition event, which is congruent with the results obtained using our first strategy (using the EVD sequence for similarity search). The other TEASV corresponded to the transposition of the CACTA transposon, CAC1 (Fig. 2). These 10 insertions were validated through wet lab analyses (Fig. 3k)

\section{Conclusion/Discussion}

In order to demonstrate the suitability of Nanopore technology for TEASV detection, we sequenced an epiRIL from $A$. thaliana. We used three runs of MinIon that in our hands produced from 38 to $232 \mathrm{Mbp}$, reaching a total of $336 \mathrm{Mb}$. This variation in yield was mostly attributed to differences in flow cell quality, according to the manufacturer's technical support. None of the flowcells provided subsequently indeed showed such discrepancies (personnal communication). After checking the quality of the sequences through homology searches against the Arabidopsis reference sequence, only $80 \mathrm{Mb}$, i.en $23 \%$ of the initial dataset could be used for further analyses. We do not provide any explanation for such large difference. Whether the non-Arabidopsis sequences originate from artefacts or from contaminants should be further tested. In addition, this result could not be attributed to the parameters used for the BLAST searches, as lowering the stringency of the searches did not modify the results. The latest flowcells and R9 chemistry developed by ONT appear to produce more reliable data. Further analyses are needed to confirm this.

The median of true Arabidopsis sequence reads was $4.6 \mathrm{~kb}$. This represents a significant technological improvement compared to other NGS platforms and even compared to the Sanger-based techniques that could not generate reads larger than 700-800 nucleotides. In this regard, our results confirm that ONT could produce data of interest for the study of structural variants. We however first had to demonstrate that the transposition of the $E V D$ retrotransposon could be detected through SRM. Using simple Blast homology searches of the reads again this TE, we were able to identify homology breakpoints and thus unambiguously map some of its insertions. Unfortunately, given the low amount of useful sequence data, we were not able to exhaustively count the number of new EVD insertions in this plant material. However, this first study leads us to conclude that despite a sequencing error rate of $13 \%$, the reliable detection of $E V D$ insertions was possible with only $0.8 \mathrm{X}$ genome coverage. Therefore, this pilot experiment constitutes a proof of concept that Nanopore technology is perfectly suitable for TEASV detection.

The real power of this new technology could only be demonstrated if it allows to identifiy exhaustively all transposition events in an individual without prior knowledge of active TE families. In the case of the Arabidopsis epiRIL that we tested, our ab initio search yielded the detection of a CACTA transposon insertion. This opens new perspective for the exhaustive characterization of active TEs in plants and animals. The identification of a $7 \mathrm{~kb}$ long read spanning a complete $E V D$ element together with both sequences flanking its insertion indeed suggests that the Nanopore technology could greatly help identify active TEs in species for which high quality genomic resources (in particular genome assembly) are not available. This will however undoubtedly require the development of new Informatic tools specially designed for the analyses of such sequence data. Our analyses relied upon the use of Blast algorithm for the ab initio detection of TEASVs. This algorithm, although fast and powerful, may not be the best suited for such purpose. In particular, the split of the alignments in multiple HSPs at multiple loci (in case the sequence flanking the insertion of the TE contains repeats) is prone to FDR. We indeed had to validate our candidates both in silico (through manual examination 


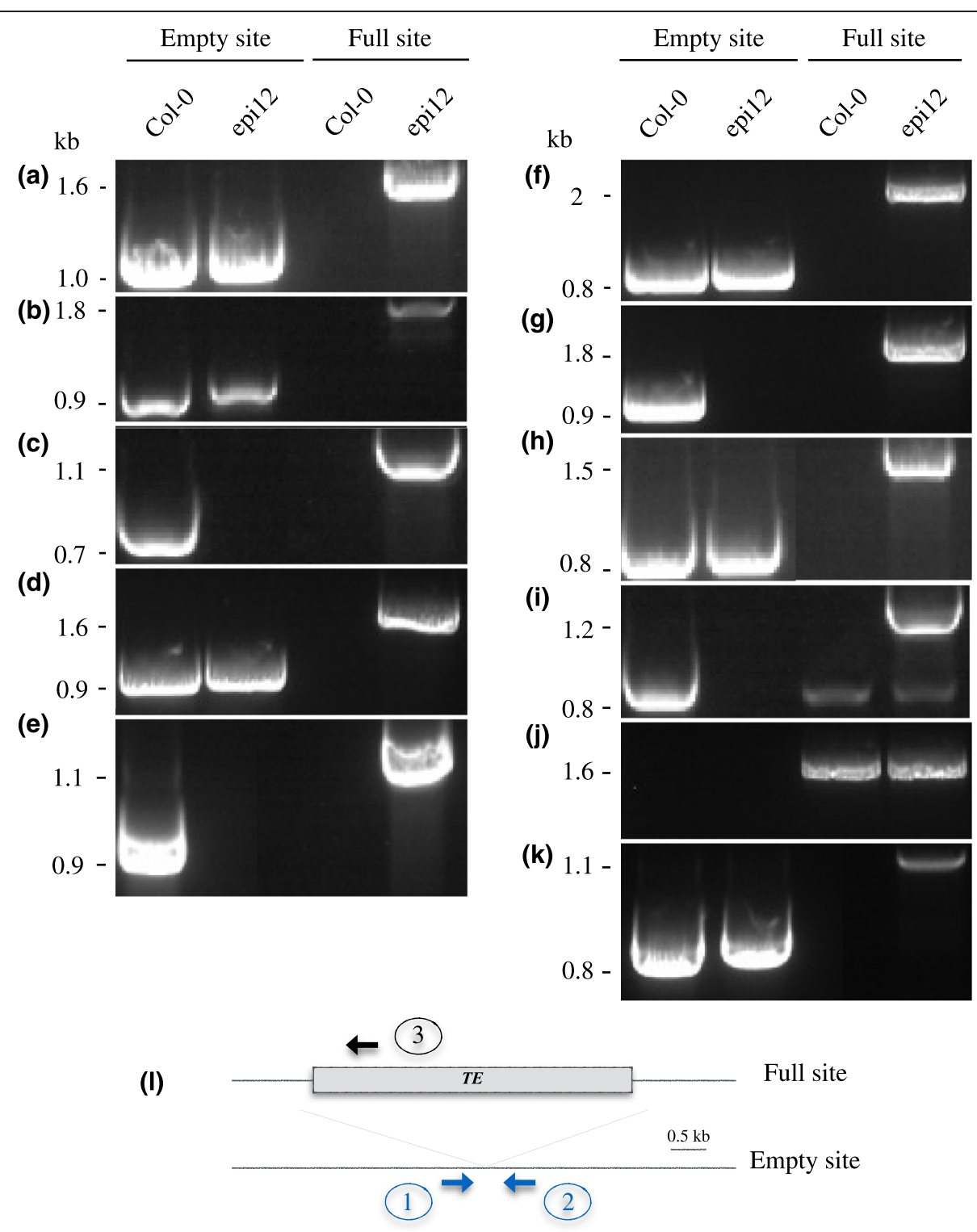

Fig. 3 Validation of EVD insertions in epiRIL12 detected by MinION. PCR detection of EVD insertion polymorphism for neo-insertion 1 to 9 (a to i), the native copy of EVD on chromosome 5 as control (j), and the neoinsertion of CAC1 ( $\mathbf{k})$. I : schematic illustration of RBIP primer design. primers 1 and 2 are designed to detect empty sites and primers 1 and 3 (sense insertion) or 3 and 2 (antisense insertion) are designed to detect full sites

of homologies) and through wet lab experiments. The use of algorithms that allow the sequence comparison of larger sequences (e.g. NucMer [22]) may considerably improve the efficiency of TEASV detection based on ONT data, although this has to be tested.

As we mentioned in the introduction, we anticipated that the trade-off between the error rate and the read length may be in favor of the latter. This is confirmed in our analysis. Although a direct comparison between ONT and Illumina platform on the same DNA source is needed to draw any conclusion on the superiority of one technology versus the other, the possibility to detect TEASVs with a $0.8 \mathrm{X}$ genome coverage is very promising, considering that, in the case of Illumina-based data, a genome coverage of at least 20X is necessary to reliably detect any TEASVs. We anticipate that a reliable identification of TEASVs could be achieved using low genome coverage, e.g. $2 \mathrm{X}$, which may contribute to keep the detection cost to a reasonable level, as long as the technology is improved in terms of sequence reliability. Additional pilot experiments are also needed to confirm that ONT may be suited for the analyses of larger, more complex genomes. In any case, the development of single molecule sequencing technologies with long reads, such as ONT, opens new perspectives in many aspects of genomics. The reliable genome-wide characterization 
of structural variations, whether at individual level (e.g. somatic variations) or within populations, will help discover some new functional aspects of genome dynamics in plant and animals.

\section{Methods}

Plant material

Arabidopsis thaliana WT ecotype Columbia-0 and epiRIL12 plant materials previously described in [23], were used in this study. Plants were grown in soil under a $16 \mathrm{~h} / 8 \mathrm{~h}$ (light/dark) cycle after 2 days at $4{ }^{\circ} \mathrm{C}$ for stratification.

\section{DNA preparation}

Genomic DNA from epiRIL12 was extracted from seedlings ground to a fine powder in liquid N2. The powder was resuspended in $10 \mathrm{ml}$ of CTAB2X extraction buffer (2\% CTAB, $100 \mathrm{mM}$ Tris- $\mathrm{HCl} \mathrm{pH} 8,20 \mathrm{mM}$ EDTA $\mathrm{pH} 8,1.4 \mathrm{M} \mathrm{NaCl}, 5 \% \mathrm{~N}$-lauroylsarcosine di- sodium salt, $0.2 \%$ 2-mercaptoethanol) and incubated for $60 \mathrm{~min}$ at $65{ }^{\circ} \mathrm{C}$. Then, an equal volume of chloroform was added and the emulsion was maintained during $10 \mathrm{~min}$ before centrifugation at 4,500 rpm for $10 \mathrm{~min}$ at room temperature. The nucleic acids were precipitated with isopropanol $(0.7 \mathrm{v} / \mathrm{v})$ at $-80{ }^{\circ} \mathrm{C}$ for $15 \mathrm{~min}$ and centrifuged at $4{ }^{\circ} \mathrm{C}$ at $4,500 \mathrm{rpm}$ for $45 \mathrm{~min}$. Nucleic acids were further washed with $75 \%$ ethanol and centrifuged at $4{ }^{\circ} \mathrm{C}$ at $4,500 \mathrm{rpm}$ for $10 \mathrm{~min}$. Finally, the pellet was air dried and DNA was resuspended in $300 \mu \mathrm{l}$ TE and was treated with $10 \mu \mathrm{g}$ of RNase A (Qiagen, Hilden, Germany) for $30 \mathrm{~min}$ at $65^{\circ} \mathrm{C}$ before further analysis.

\section{Estimation of EVD copy number}

DNA was quantified using the Quant-iT dsDNA High sensitivity Assay (HS) Kit (Life Technologies) according to the manufacturer's instructions. For qPCR analysis of EVD DNA copies, the Takyon No RoxSYBR MasterMix dTTP Blue Kit (Eurogentec, Liège, Belgium) was used and ACTIN2 was used to normalize DNA levels. DNA copy number was performed in a final volume of $10 \mu \mathrm{l}$ employing a LightCycler ${ }^{\circ} 480$ (Roche, Basel, Switzerland). PCR were performed with primers summarize in Additional file 3: Table S1 with the following conditions: $95{ }^{\circ} \mathrm{C}$ for $5 \mathrm{~min}$ followed by 45 cycles of amplification composed of $10 \mathrm{~s}$ at $95{ }^{\circ} \mathrm{C}, 10 \mathrm{~s}$ at $60{ }^{\circ} \mathrm{C}$ and $10 \mathrm{~s}$ at $72{ }^{\circ} \mathrm{C}$, then a final soak at $37^{\circ} \mathrm{C}$ for $30 \mathrm{~s}$ was done. The EVD DNA copies were determined by the $2 \Delta \Delta \mathrm{CT}$ method and diagram represent relative quantity of amplification compared to the WT, which was taken as 2. Taking into account that the presence of extrachromosomal forms of the element may induce a strong bias in the estimation of new EVD copies, we estimate that the line harbours at most 13 new insertions (Additional file 4: Figure S1).

\section{Minlon library preparation}

The MinIon sequencing library was generated with the sequencing Kit (SQK-MAP-006) (Oxford Nanopore Technologies, Oxford, UK) according to the manufacturer's instructions. Four microgram of DNA were fragmented as follows: DNA was loaded into a G-tube (Covaris, Brighton, UK) and spun at 6,000 rpm in an eppendorf 5424 for $1 \mathrm{~min}$ before inverting the tube to centrifuge again for $1 \mathrm{~min}$. One microgram of fragmented DNA was end-repaired and dA-tailed using NEBNext Ultra II end-repair/dA-tailing module (Biolabs, New England, USA, cat. no. E7546S) as per the manufacturer's instructions except for the thermal cycler program performed with $5 \mathrm{~min}$ at $20^{\circ} \mathrm{C}$, followed by $5 \mathrm{~min}$ at $65{ }^{\circ} \mathrm{C}$ and terminated with $5 \mathrm{~min}$ at $4{ }^{\circ} \mathrm{C}$. The DNA was further purified with 1.0X vol. Agencourt AMPure XP beads (Beckman Coulter, High Wycombe, UK). After two washes with $70 \%$ of ethanol, beads were air dried and the DNA was elutated with $31 \mu \mathrm{l}$ of Gibco distilled water. A ligation with biotinylated hairpin adapters was then performed by adding $50 \mu \mathrm{l}$ Blunt/TA ligase master MIX (Biolabs, New England, US, cat. no. M0367S) to the A-tailed library. Library was enriched of molecules fixed with biotinylated hairpin thanks to the use of MyOneTM Streptavidin C1 Dynabeads (Thermofisher) prepared with bead binding buffer (BBB). Then, library was eluted off beads using $25 \mu \mathrm{l}$ of elution buffer (ELB). The DNA concentration in the final library was quantified using a Quant-iT.

\section{Sequencing}

Three Oxford Nanopore flow cells: 1 FLO-MAP003 and 2 FLO-MAP103 were used in this study. The number of available pores was first recorded to evaluate the flow cell's quality with the MinKNOW ${ }^{\mathrm{m}}$ software. The version v0.51.1.62 of MinKNOW ${ }^{\mathrm{ma}}$ software was used with the flow cell FLO-MAP003 and version v0.51.3.40 with the two other flow cells FLO-MAP103. Before starting the analyses, the Minknown application was first used to make the platform QC before starting the analyses and a map/NC-48-h sequencing run flow (Map 103) protocol was chosen and initiated. $500 \mu \mathrm{l}$ of a priming mix (26.6 $\mu \mathrm{l}$ of Fuel mix (FMX), $500 \mu \mathrm{l}$ of Running buffer (RNB), 473.4 $\mu \mathrm{l}$ of Nuclease-free water) were introduced two times in the MinIon flow cell to primed the sensory array. Thirty-six ng of the prepared library was diluted into a mix composed of $75 \mu \mathrm{l}$ of RNB, $4 \mu \mathrm{l}$ of FMX and Nuclease-free water was added to reach a final volume of $150 \mu \mathrm{l}$. The $150 \mu \mathrm{l}$ priming mix was loaded into the sample loading port of the flow cell. The sequencing reaction was started. After 24 hours, $150 \mu \mathrm{l}$ of a priming mix with $36 \mathrm{ng}$ of the library was loaded again. One of the flow-cells FLO-MAP103 was loaded with a priming mix containing $108 \mathrm{ng}$ of the prepared library. Base- 
calling was performed through data transference using the Metrichor $^{\mathrm{Tm}}$ agent (v2.39.3) and 2D base-calling workflow (v1.69). During the sequencing run, one additional freshly diluted aliquot of DNA library was loaded after $24 \mathrm{~h}$ of initial input.

\section{Characterisation of sequencing raw data}

Raw reads were first extracted from the native HDF5 format MinIon reads using poretools [24] (Loman and Quinlan, 2014). Similarities between each read and Arabidopsis genome were estimated using a BlastN search using standard parameters, the read as query and the TAIR10 version of genome assembly as database. Reads producing alignment on TAIR10 assembly on at least $20 \%$ of their length were kept for further analyses. The estimation of the percentage of identity between the reads and the Arabidopsis genome was achieved by averaging that of all HSPs.

\section{Detection of EVD insertions}

A BLAST multifasta database of all the MinIon reads was created with formatdb (version 2.2.26). Then the EVD sequence (ATCOPIA93, [11]) was aligned against the multifasta database with blastall (version 2.2.26) with the default parameters. For each blast hit, a dotter (version 4.1) with the Nanopore read against the $E V D$ sequence was produced. In parallel, the same Nanopore read was aligned against the Arabidopsis thaliana genome, using ncbi BLAST with default parameters in order to identify the position of the flanking region. The start and end position of the alignement were kept and a sequence with plus or minus $1 \mathrm{~kb}$ was extracted from the Arabidopsis thaliana genome for each position. The extracted sequence and the $E V D$ sequence were concatenated and a dotter with the 2D fasta MinIon read and the concatenated sequence was performed. Only the reads showing a clear similarity with the EVD and the flanking sequence, based on visual examination of the dotter output were kept for further characterization.

\section{ab initio detection}

The ab initio detection method that we developed is conceptualy close to the Split Read Method developped for Illumina data, except that the long reads allow a systematic use of Blast algorithm. First, each read was aligned against TAIR10 genome assembly using BlastN using the -G 5 -E 2 -r 2 parameters in order to take into account the error rate generated by the technology. Those for which a maximum of $75 \%$ and a minimum of $400 \mathrm{bp}$ contiguous sequences could be aligned were kept as putative representative of similarity breakpoints and then aligned against the Arabidopsis TE database (www.arabidopsis.org). Only the reads for which at least
$200 \mathrm{bp}$ of the remaining portion could be aligned on a well characterized TE were selected. After these filtering steps, each positive read was again compared to the putative region of the TE insertion (obtained by performing an extractseq of a $2 \mathrm{~kb}$ region spanning the insertion site) using the blast2seq software. Finally, only reads for which the sum of HSP length was higher than $300 \mathrm{bp}$ and comprised between 33 and $75 \%$ of the total read length were kept. These candidates were finally checked manually using of dotter visualization (similarly to the procedure used for the identification of $E V D$ insertions described above).

\section{Wet-lab validations}

RBIP is a PCR-based marker strategy using a threeprimer set derived from the flanking region and the LTR to the LTR-RT TE. Here, RBIP primer sets were designed to amplify the full sites between the internal TE sequence and its flanking sequence. Primers used to confirm the point of neo-insertion were designed with the Primer3 tool http://primer3.ut.ee/, 0.4.0 version [25] (Additional file 3: Figure S3). PCR was performed in a volume of $15 \mu \mathrm{l}$ containing $3 \mu \mathrm{l}$ of Gotaq ${ }^{\bullet}$ DNA polymerase Buffer (Promega, Madison, WI, USA), $8.05 \mu \mathrm{l}$ water, $0.3 \mu \mathrm{l}$ of $10 \mu \mathrm{M}$ dNTPs , $0.5 \mu \mathrm{l}$ of $10 \mu \mathrm{M}$ of each primer, $0.75 \mathrm{U}\left(0.15 \mu \mathrm{l}\right.$ of Gotaq $^{\circ}$ DNA polymerase and $2.5 \mu \mathrm{l}$ of $1 \mathrm{ng} / \mu \mathrm{l}$ DNA. Amplification was conducted under the same parameters except for the elongation step depending of the expected amplicon size. Regions containing the entire $E V D$ insertion was amplified with an elongation time of $5 \mathrm{~min}$. All other parameters were identical to the classical PCR approach. The amplification was performed with the program described as follows: Initial hot start $\left(3 \mathrm{~min}, 94{ }^{\circ} \mathrm{C}\right)$ and then 30 cycles with denaturation $\left(30 \mathrm{~s}, 94{ }^{\circ} \mathrm{C}\right)$, annealing $\left(30 \mathrm{~s}, 62{ }^{\circ} \mathrm{C}\right)$, elongation (1-5 min, $\left.72{ }^{\circ} \mathrm{C}\right)$, with a final soak (4 min, $\left.20{ }^{\circ} \mathrm{C}\right)$. The amplified product was electrophoresed at $100 \mathrm{~V}$ on a $1 \%$ agarose gel (Euromedex, Souffelweyersheim, France) in a 0.5X TAE buffer $(20 \mathrm{mM}$ Tris, $10 \mathrm{mM}$ acetate, $1 \mathrm{mM}$ EDTA pH 8.3). The $1 \%$ agarose gel solution was prepared with Gel redTM 10,000X stock reagent (Interchim, Montluçon, France) diluted at 1:10,000. PCR amplicons were purified with the Geneclean purification kit (MP Biomedicals, Santa Ana, CA, USA) according to the manufacturer's instructions. Sanger sequencing was performed with the same primers used to obtain these PCR amplicons on the ABI3130X1 Genetic Analyser sequencer (Applied Biosystems, Thermo Scientific, Waltham, MA, USA) at the Sequencing platform of the LGDP, Perpignan, France. Analysis were then performed using the Ridom TraceEdit software (Ridom Bioinformatics, Würzburg, Germany). 


\section{Additional files}

Additional file 1 Figure S2. Box-plot of MinlON read length distribution. (PDF $24 \mathrm{~kb}$ )

Additional file $\mathbf{2}$ S3 text file. Genomic sequences of 10 unique loci used to estimate the genome coverage of sequence data. Sequences are given in fasta format. (TXT $40 \mathrm{~kb}$ )

Additional file $\mathbf{3}$ Table S1. Primers used for quantitative PCR and for real-time PCR. (XLS 64 kb)

Additional file 4 Figure $\mathbf{S 1}$. Accumulation of EVD DNA. DNA

accumulation of EVD for wild type (WT) and epiRIL12 (epi12) measured by qPCR (mean \pm s.e.m., $n=3$ technical repetitions). (PDF $16 \mathrm{~kb}$ )

\section{Abbreviations}

DOC: Depth Of Coverage.; EpiRIL: Epigenetic Recombinant Imbred Line.; EVD: Evadé.; FDR: False discovery rate.; LTR: Long Terminal Repeat:; MULE: Mu-Like Element.; NGS: Next generation sequencing.; ONT: Oxford Nanopore Technology.; PCR: Polymerase Chain Reaction.; PEM: Paired End Mapping.; SRM: Split Read Mapping.; TE: Transposable Element.;

TEASV: Transposable Elements-associated Structural Variants.

\section{Acknowledgements}

The authors thank Jerzy Paszkowski and Jon Reinders for providing seeds of the epiRIL.

\section{Funding}

Emilie DEBLADIS is supported by a grant from the french Ministry of Education. This work was supported by a BQR grant from the University of Perpignan Via Domitia.

\section{Availability of data and materials}

The fasta files of all Nanopore reads from the 3 Minlon runs are available at http://gamay.univ-perp.fr/ Panaudlab/EpiR_all_seq.fasta.tar.gz. Seeds of the epi-RIL12 used in this study are available upon request.

\section{Authors' contribution}

ED designed the experiments, conducted the analyses and wrote the manuscript. $C L$ performed the library preparation and the sequencing. M-CC provided support for the bioinformatic analyses. MM provided support for the characterization of plant material. OP supervised the work.

\section{Competing interests}

None of the authors have competing interest.

\section{Consent for publication}

Not applicable.

\section{Ethics approval and consent to participate}

The plant material used in this study is a line derived from a cros between the wild type Col0 (an accession of the species Arabidopsis thaliana used worldwide as a reference for plant research) and a mutant line impeded in methylation pathway. There is no ethics issue with this material.

\section{Publisher's Note}

Springer Nature remains neutral with regard to jurisdictional claims in published maps and institutional affiliations.

\section{Author details}

'Université de Perpignan Via Domitia, Laboratoire Génome et Développement des Plantes, 52, avenue Paul alduy, 66860 Perpignan cedex, France. ${ }^{2}$ Centre National de la Recherche Scientifique, Laboratoire Génome et Développement des Plantes, 52, avenue Paul alduy, 66860 Perpignan cedex, France. ${ }^{3}$ Institut de Recherche pour le Développement, UMR232 DIADE Diversité Adaptation et Développement des Plantes, Perpignan, France. ${ }^{4}$ Institut Universitaire de France, Paris, France.
Received: 28 November 2016 Accepted: 3 May 2017

Published online: 17 July 2017

\section{References}

1. Britten RJ, Kohne DE. Repeated sequences in DNA. Science. 1968;161:529-40.

2. Feschotte C, Pritham EJ. DNA transposons and the evolution of eukaryotic genomes. Annu RevGenet. 2007:41:331-68.

3. Lisch D. How important are transposons for plant evolution? Nat Rev Genet 2013;14:49-61.

4. Zhao D, Ferguson AA, Jiang N. What makes up plant genomes: The vanishing line between transposable elements and genes. Biochim Biophys Acta. 1859;2016:336-80.

5. Kobayashi S, Goto-Yamamoto N, Hirochika H. Retrotransposon-induced mutations in grape skin color. Science. 2004;304:982.

6. Wei L, Cao X. The effect of transposable elements on phenotypic variation: insights from plants to humans. Sri China Life Sci. 2016;59:24-37.

7. Makalowski W, Pande A, Gotea V, Makalowska I. Transposable elements and their identification. Methods Mol Biol. 2012;855:337-59.

8. Ewing AD. Transposable element detection from whole genome sequence data. Mob DNA. 2015:6:24

9. Sabot F, Picault N, El-Baidouri M, Llauro C, Chaparro C, Piegu B, et al. Transpositional landscape of the rice genome revealed by paired-end mapping of high-throughput re-sequencing data. Plant J. 2011;66:241-6.

10. Cheng C, Tarutani Y, Miyao A, Ito T, Yamazaki M, Sakai H, et al. Loss of function mutations in the rice chromomethylase OsCMT3a cause a burst of transposition. Plant J. 2015:83:1069-81.

11. Mirouze M, Reinders J, Bucher E, Nishimura T, Schneeberger K, Ossowski S, et al. Selective epigenetic control of retrotransposition in Arabidopsis. Nature. 2009:461:427-30

12. Handsaker RE, Kom JM, Nemesh J, McCarroll SA. Discovery and genotyping of genome structural polymorphism by sequencing on a population scale. Nat Genet. 2011;43:269-76.

13. Mills RE, Walker K, Stewart C, Handsaker RE, Chen K, Alkan C, et al. Mapping copy number variation by population-scale genome sequencing. Nature. 2011:470:59-65.

14. Medvedev P, Stanciu M, Brudno M. Computational methods for discovering structural variation with next-generation sequencing. Nat Methods. 2009:6: S13-20

15. Ye K, Schulz MH, Long $Q$, Apweiler $R$, Ning Z. Pindel: a pattern growth approach to detect break points of large deletions and medium sized insertions from paired-end short reads. Bioinformatics. 2009:25:2865-71.

16. Yoon S, Xuan Z, Makarov V, Ye K, Sebat J. Sensitive and accurate detection of copy number variants using read depth of coverage. Genome Res. 2009; 19:1586-92

17. Glusman G, Severson A, Dhankani V, Robinson M, Farrah T, Mauldin DE, et al. Identification of copy number variants in whole-genome data using Reference Coverage Profiles. Front Genet. 2015;6:45.

18. Quadrana L, Bortolini Silveira A, Mathew GF, LeBlanc C, Martienssen RA Jeddeloh JA, et al. The Arabidopsis thaliana mobilome and its impact at the species level. elife. 2016:5:e15716.

19. Deamer $D$, Akeson M, Branton D. Three decades of nano pore sequencing. Nat Biotechnol. 2016;34:518-24.

20. Feng Y, Zhang Y, Ying C, Wang D, Du C. Nanopore-based fourth-generation DNA sequencing technology. Genomics, Proteomics Bioinformatics. 2015;13: 4-16.

21. Laver T, Harrison J, O'Neill PA, Moore K, Farbos A, Paszkiewicz K, et al. Assessing the performance of the Oxford Nanopore Technologies Minlon. Biomol Detect Quantif. 2015;3:1-8.

22. Kurtz S, Phillippy A, Delcher AL, Smoot M, Shumway M, Antonescu C Salzberg SL. Versatile and open software for comparing large genomes. Genome Biol. 2004:5(2):R12.

23. Reinders J, Wulff BBH, Mirouze M, Marí-Ordóñez A, Dapp M, Rozhon W, et al. Compromised stability of DNA methylation and transposon immobilization in mosaic Arabidopsis epigenomes. Genes Dev. 2009;23:939-50.

24. Loman NJ, Quinlan AR. Poretools: a toolkit for analyzing nanopore sequence data. Bioinformatics. 2014:30:3399-401.

25. Koressaar T, Remm M. Enhancements and modifications of primer design program Primer3. Bioinformatics. 2007:23:1289-91. 